\title{
People's Opinion, People's Nexus, People's Security and Computational Intelligence: The Evolution Continues
}

\author{
Ali Ghorbani \\ Faculty of Computer Science, University of New Bunswick \\ Box 4400 Fredericton, N.B., Canada \\ ghorbani@unb.ca
}

The talk begins with a brief introduction to some of our research work in the past few years as well as the ongoing research. A new model on extending the flexibility and responsiveness of websites through automated learning for customtailoring and adaptive web to user usage patterns, interests, goals, knowledge and preferences will be presented. The second part of the talk will be devoted to the challenges that the Computational Intelligence communities are faced with in order to address issues related to people's nexus, opinion, and security on the Web, and our contributions to these topics. At the end, I will provide an overview of our current research focus on network security and intelligence information handling and disimination. 